\title{
Global Multivariable Control of Permanent Magnet Synchronous Motor for Mechanical Elastic Energy Storage System under Multiclass Nonharmonic External Disturbances
}

\author{
Yang Yu, Zengqiang Mi, Yikun Xu, and Tong Zhao \\ State Key Laboratory of Alternate Electrical Power System with Renewable Energy Sources, North China Electric Power University, \\ Baoding 071003, China \\ Correspondence should be addressed to Yang Yu; ncepu_yy@163.com
}

Received 4 November 2013; Accepted 28 April 2014; Published 26 May 2014

Academic Editor: Shuping He

Copyright (c) 2014 Yang Yu et al. This is an open access article distributed under the Creative Commons Attribution License, which permits unrestricted use, distribution, and reproduction in any medium, provided the original work is properly cited.

\begin{abstract}
For the technology of mechanical elastic energy storage utilizing spiral torsion springs as the energy storage media presented previously, a global multivariable control algorithm based on nonlinear internal model principle under multiclass external disturbances is proposed. The nonlinear external disturbances with nonharmonic periodic characteristics are generated by multiclass nonlinear external systems. New equations of nonlinear internal model are designed to estimate the multiclass external disturbances. On the basis of constructing the control law of nominal system, a state feedback controller is designed to guarantee the closed-loop system globally uniformly bounded, and a Lyapunov function is constructed to theoretically prove the global uniform boundedness of the multivariable closed-loop system signals. The simulation results verify the correctness and effectiveness of the presented algorithm.
\end{abstract}

\section{Introduction}

Energy storage technologies have a great practical significance for the solution of new energy interconnecting to the grid, peak regulation, frequency modulation, and stability control $[1,2]$. Based on lucubration of mechanical elastic energy storage (MEES), a new way of MEES method applying spiral torsion springs (STS) as the energy storage material is proposed [3]. Due to the advantages such as large power density, high efficiency, great electromagnetic torque, small volume, and fast response speed, permanent magnet synchronous motor (PMSM) is selected as the actuator for MEES system, just as in many other servo systems [4]. One of the key technologies to be solved for MEES is the nonlinear control owing to its electromechanical coupling properties and nonlinear characteristics of PMSM [5]. When PMSM based MEES system runs in energy storage, the increasing load torque with the tightening of STS is unfavorable for the operation of servo system. In addition, due to the nonlinearities, strong coupling, and time variation of PMSM, especially the existence of the nonlinear external disturbances, the conventional PID controller is difficult to satisfy the requirement of high precision control [6]. Hence, the other control methods should be introduced, just as nonlinear control [7-10], adaptive control [11], state feedback control [12], and so forth.

One of the core questions in control field is to guarantee asymptotical stability of unforced close-loop system, implement the asymptotical tracking of system output for given trajectories, and reject exogenous disturbances [13-16]. The control problems for servo system are also called the output regulation problems; the problems of disturbance rejection under the framework of output regulation have earned extensive attention in recent years $[17,18]$. The preexisting papers mostly assumed that the exosystem generating disturbances is linear, neutral, and stable; that is to say, disturbance rejection under sinusoidal perturbation is frequently studied. For instance, literatures $[16,17,19]$ deal with the problems 
of sinusoidal perturbation rejection in known and unknown frequency, respectively. Nevertheless, the nonharmonic disturbances generated by nonlinear exosystems, which will make practical servo systems, generators, and power flexible mechanisms produce noise and precision reduction [20], are harmful to these practical running systems. However, how to tackle with disturbances generated by nonlinear exosystems is rarely involved [21]. Therefore, rejection of these harmful oscillations is essential to guarantee that the systems operate stably under nonlinear external disturbances.

Another central issue in control field is to extend the established control theory to more complex and generalized circumstances. In [22], disturbance rejection under a class of exosystems for a single-input nonlinear system is studied, in which the dependent external system is a single model, and the researched system is a single-input system. In [23], a global harmonic rejection algorithm for multivariable system is proposed; however, the rejection of the disturbances in the paper aims at the standard sinusoidal components. For the nonlinearity and complexity of PMSM based MEES system, the focus of the paper is to extend the previous works in $[22,23]$ to a generalized multivariable input field of nonlinear systems under multiclass nonlinear exosystems with the application of the constructed model for PMSM based MEES system in [5] and design a controller to suppress multiclass external nonharmonic disturbances in PSMS based MEES system.

The mainly theoretical contribution of the paper is to propose a global multivariable disturbance control method to reject multiclass nonlinear external nonharmonic disturbances generated by multiclass nonlinear exosystems for general multivariable nonlinear system, and the presented control algorithm is employed to regulate an actual nonlinear system. The validity and effectiveness of the proposed method are testified by the simulation results.

The organization of the paper is as follows. It starts with an introduction of the research status of disturbance rejection and points out the significance of rejection of nonharmonic external disturbances in Section 1. The mathematical model of PMSM based MEES system is constructed and the formulation of control problem in the paper is given in Section 2. In Section 3, the nonlinear internal models simulating the multiclass external nonharmonic disturbances are presented based on internal model principle. Nonlinear multivariable state feedback controller ensuring the closedloop system globally bounded is demonstrated in Section 4 . The verifications of the proposed algorithm by means of numerical simulations are shown in Section 5. Ultimately, Section 6 sums up the conclusions of the research and puts forward the work in the future.

\section{Problem Formulation}

2.1. Mathematical Model of PMSM Based MEES System. For the convenience of understanding and reutilization, the model of the whole system for PMSM based MEES system proposed in [3,5] is shown in Figure 1, where gear case is simplified as a model of spring mass damper with multidegree

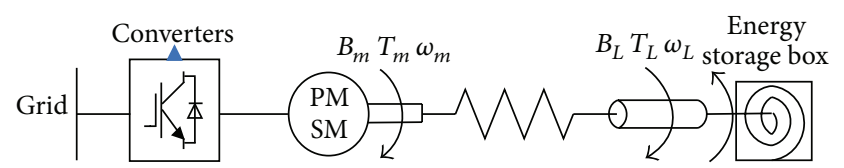

FIGURE 1: The model of PMSM based MEES system.

of freedom, $B_{m}$ and $B_{L}$ denote the damping coefficients of the motor and elastic shaft, respectively, $T_{m}$ and $T_{L}$ represent the output torque of the motor and main shaft, respectively, and $\omega_{m}$ and $\omega_{L}$ symbolize the angular velocity of the motor and main shaft of energy storage box, respectively. STS is installed in energy storage box.

In the process of energy storage, PMSM runs in the state of electric motor. For PMSM, assume that the inductance of $d$-axis $L_{d}$ is equal to the inductance of $q$-axis $L_{q}$. Accordingly, the mathematical model for PMSM in $d q$ rotating coordinates can be expressed as follows [24]:

$$
\begin{gathered}
\frac{d i_{d}}{d t}=-\frac{R_{s}}{L_{d}} i_{d}+p i_{q} \omega_{m}+\frac{1}{L_{d}} u_{d}, \\
\frac{d i_{q}}{d t}=-\frac{R_{s}}{L_{q}} i_{q}-p i_{d} \omega_{m}-\frac{p \varphi_{f}}{L_{q}} \omega_{m}+\frac{1}{L_{q}} u_{q} \\
\frac{d \omega_{m}}{d t}=\frac{p \varphi_{f}}{J_{m}} i_{q}-\frac{B_{m}}{J_{m}} \omega_{m}-\frac{1}{J_{m}} T_{m},
\end{gathered}
$$

where $i_{d}$ and $i_{q}$ denote the current components of stator in $d q$ axis, respectively, $u_{d}$ and $u_{q}$ display the voltage components of stator in $d q$ axis, respectively, and $R_{s}, \omega_{m}, \varphi_{f}, p$, and $J_{m}$ represent the resistance of stator, angular velocity, flux linkage of rotor, numbers of pole pairs, and moment of inertia of rotor, respectively.

The ratio of gear case is assumed to be $r$; without regard to the power loss of gear case, the relationship between torque and angular velocity on both sides of gear case can be expressed as

$$
T_{m}=\frac{T_{L}}{r}, \quad \omega_{m}=\omega_{L} \cdot r .
$$

Suppose the outer end of STS to be fixed in spring box as $\mathrm{V}$ type, and in terms of the national standard design and calculation of spiral torsion spring (JB/T7366-1994) in China, the torque of STS with rectangle cross section can be written as

$$
T_{L}=k \frac{E b h^{3}}{6 l} n,
$$

where $n$ denotes the working turns of spring, $E, l, b$, and $h$ represent the modulus of elasticity, length, width, and thickness of STS, respectively, and $k$ indicates mass coefficient of spring.

Due to the large mass and high inertia of MEES system, the working rotation velocities for the main shaft of energy storage box and the rotor of PMSM are both assumed to be invariable. Hence, the relationship between the angular 
velocity $\omega_{L}$ and the working turns $n$ for the main shaft of energy storage box can be written as follows:

$$
n=\frac{\omega_{L} t}{2 \pi}
$$

where $t$ represents the time.

Equation (4) is substituted into (3); the relationship between the torque $T_{L}$ of STS and angular velocity $\omega_{L}$ of the main shaft can be given by the following formula:

$$
T_{L}=k \frac{E b h^{3} \omega_{L}}{12 \pi l} t
$$

For a given STS, (5) shows that the torque $T_{L}$ of STS is proportional to the time $t$ if angular velocity $\omega_{L}$ is a constant.

The mathematical model for the whole system of PMSM based MEES can be obtained by combining differential equation (1) with algebraic equations (2) and (5).

2.2. Control Problem Formulation. Consider the multivariable nonlinear system with a standard affine form under multiclass disturbances:

$$
\dot{\mathbf{x}}=\mathbf{f}(\mathbf{x})+\sum_{i=1}^{m} \mathbf{g}_{i}(\mathbf{x})\left(\mathbf{u}_{i}-\mathbf{v}_{i}(\mathbf{w})\right), \quad 1 \leq i \leq m,
$$

where $\mathbf{x} \in \mathbf{R}^{n}$ are the state vectors, $\mathbf{u}_{i} \in \mathbf{R}$ are the control inputs, $\mathbf{f}(\mathbf{x})$ and $\mathbf{g}_{i}(\mathbf{x})$ are the known smooth vector fields, $\mathbf{v}_{i}(\mathbf{w})$ are the nonlinear disturbance inputs, and $\mathbf{w} \epsilon$ $\mathbf{R}^{q}$ indicate the external signals generated by the nonlinear exosystem shown as follows:

$$
\dot{\mathbf{w}}=\mathbf{s}_{i}(\mathbf{w}), \quad 1 \leq i \leq m .
$$

If the nonlinear disturbance inputs are ignored, for system (6), its nominal system can be written as

$$
\dot{\mathbf{x}}=\mathbf{f}(\mathbf{x})+\sum_{i=1}^{m} \mathbf{g}_{i}(\mathbf{x}) \mathbf{u}_{i}, \quad 1 \leq i \leq m
$$

The essence of solving stability problems for a multivariable input system is to convert these problems into the stability problems of multiple single-input systems [25].

Assumption 1. For system (8), there exists a control law of state feedback $\boldsymbol{\alpha}_{i}(\mathbf{x})$ making the nominal close-loop system $\dot{\mathbf{x}}=\mathbf{f}(\mathbf{x})+\sum_{i=1}^{m} \mathbf{g}_{i}(\mathbf{x}) \boldsymbol{\alpha}_{i}(\mathbf{x})$ asymptotically stabilize at the origin. Therefore, there exists a Lyapunov function $\mathbf{V}(\mathbf{x})$ satisfying

$$
\begin{gathered}
\underline{d}(\|\mathbf{x}\|) \leq \mathbf{V}(\mathbf{x}) \leq \bar{d}(\|\mathbf{x}\|) \\
\frac{\partial \mathbf{V}(\mathbf{x})}{\partial \mathbf{x}}\left(\mathbf{f}(\mathbf{x})+\sum_{i=1}^{m} \mathbf{g}_{i}(\mathbf{x}) \boldsymbol{\alpha}_{i}(\mathbf{x})\right) \leq-d_{0}(\|\mathbf{x}\|), \\
\left|\frac{\partial \mathbf{V}(\mathbf{x})}{\partial \mathbf{x}} \sum_{i=1}^{m} \mathbf{g}_{i}(\mathbf{x})\right|^{2} \leq d_{0}(\|\mathbf{x}\|)
\end{gathered}
$$

where $\underline{d}, \bar{d}$, and $d_{0}$ are all of $K_{\infty}$ class functions.
Assumption 2. The trajectory of the vector field for the nonlinear exosystem (7) is bounded.

Remark 3. The functions, which meet Assumption 2, include harmonic functions and limit cycles of nonlinear dynamic systems. For instance, the famous Van der Pol circuit can be written as

$$
\begin{aligned}
& \dot{w}_{1}=w_{2}-\varsigma\left(\frac{1}{3} w_{1}^{3}-w_{1}\right), \\
& \dot{w}_{2}=-w_{1},
\end{aligned}
$$

where $\varsigma$ denotes a parameter to adjust the period of current or voltage. The eigenvalues of Jacobian matrix for (10) at the origin are $(1 / 2)\left(\varsigma \pm \sqrt{\varsigma^{2}-4}\right)$. If $0<\varsigma \leq 2$, the eigenvalues have positive real parts; if $\varsigma>2$, the eigenvalues are positive real numbers. Consequently, as long as $\varsigma>0$, the equilibrium points at the origin of (10) are unstable and there exists a bounded limit cycle [20].

Assumption 4. There exists a smooth function $\mathbf{r}_{i}(\mathbf{x}): \mathbf{R}^{n} \rightarrow$ $\mathbf{R}^{q}$ making

$$
\frac{\partial \mathbf{r}_{i}(\mathbf{x})}{\partial \mathbf{x}} \mathbf{g}_{i}(\mathbf{x})=\mathbf{K}_{i}, \quad 1 \leq i \leq m,
$$

where $K_{i} \in \mathbf{R}^{q}$ is a nonzero constant vector.

The problem to be solved in the paper can be described as in the following definition.

Definition 5. For any given compact subset $\mathbf{D}_{\mathbf{w}} \in \mathbf{R}^{q}$, a state feedback controller $\mathbf{u}_{i}$ can be found to make the solution of the close-loop system (6) exist and be bounded, and $\lim _{t \rightarrow \infty} \mathbf{x}(t)=0$ under arbitrary initial conditions for all $\mathbf{w}(\mathbf{0}) \in \mathbf{D}_{\mathbf{w}}$ and $t \geq 0$.

\section{Multiclass Nonlinear Internal Models Design}

In the paper, internal model principle (IMC) is utilized to reject the multiclass disturbances. Disturbances rejection by IMC belongs to indirect suppression algorithm. Hence, appropriate internal model equations should be firstly established to estimate the inputting nonlinear disturbances. Because the exosystem discussed in the paper is nonlinear, the internal model equations established should also be nonlinear. Therefore, Assumption 6 is introduced as follows.

Assumption 6. For the nonlinear exosystem (7), when $1 \leq$ $i \leq m$, there exists an immersion system being depicted as follows:

$$
\dot{\boldsymbol{\eta}}_{i}=\mathbf{F}_{i} \boldsymbol{\eta}_{i}+\mathbf{G}_{i} \gamma_{i}\left(\mathbf{J}_{i} \boldsymbol{\eta}_{i}\right), \quad \mathbf{v}_{i}(\mathbf{w})=\mathbf{H}_{i} \boldsymbol{\eta}_{i},
$$

where $\boldsymbol{\eta}_{i} \in \mathbf{R}^{r}, \mathbf{F}_{i}, \mathbf{G}_{i}, \mathbf{H}_{i}, \mathbf{J}_{i}$ are matrices with certain dimensions, the matrix pair $\left(\mathbf{F}_{i}, \mathbf{H}_{i}\right)$ is observable, and there exists a positive definite matrix $\mathbf{P}_{\widehat{\boldsymbol{\eta}}_{i}}$ making the following formula hold:

$$
\mathbf{P}_{\widehat{\boldsymbol{\eta}}_{i}} \mathbf{G}_{i}+\left(\mathbf{J}_{i}\right)^{T}=0
$$


and the nonlinear function $\gamma_{i}\left(\mathbf{J}_{i} \boldsymbol{\eta}_{i}\right)$ can be expressed as

$$
\gamma_{i}\left(\mathbf{J}_{i} \boldsymbol{\eta}_{i}\right)=\left[\begin{array}{c}
\gamma_{i}^{1}\left(\sum_{j=1}^{r} \mathbf{J}_{i}^{1 j} \boldsymbol{\eta}_{i}^{1 j}\right) \\
\vdots \\
\gamma_{i}^{m}\left(\sum_{j=1}^{r} \mathbf{J}_{i}^{m j} \boldsymbol{\eta}_{i}^{m j}\right)
\end{array}\right]
$$

and satisfies $\left(s_{1}-s_{2}\right)^{T}\left(\gamma_{i}\left(s_{1}\right)-\gamma_{i}\left(s_{2}\right)\right) \geq 0$.

Consequently, the multiclass nonlinear internal model equations can be designed as follows:

$$
\begin{aligned}
\dot{\hat{\boldsymbol{\eta}}}_{i}= & \left(\mathbf{F}_{i}-\mathbf{K}_{i} \mathbf{H}_{i}\right)\left(\widehat{\boldsymbol{\eta}}_{i}-\mathbf{r}_{i}(\mathbf{x})\right)+\mathbf{G}_{i} \gamma_{i}\left(\mathbf{J}_{i}\left(\widehat{\boldsymbol{\eta}}_{i}-\mathbf{r}_{i}(\mathbf{x})\right)\right) \\
& +\mathbf{K}_{i} \mathbf{u}_{i}+\frac{\partial \mathbf{r}_{i}(\mathbf{x})}{\partial \mathbf{x}} \mathbf{f}_{i}(\mathbf{x}),
\end{aligned}
$$

where $\mathbf{K}_{i} \in \mathbf{R}^{q}$ satisfies Assumption 4 and makes $\mathbf{F}_{i 0}=$ $\mathbf{F}_{i}-\mathbf{K}_{i} \mathbf{H}_{i}$ a Hurwitz matrix; hence there exist positive definite matrices $\mathbf{P}_{\widehat{\boldsymbol{\eta}}_{i}}$ and $\mathbf{Q}_{\widehat{\boldsymbol{\eta}}_{i}}$ satisfying the following equation:

$$
\mathbf{P}_{\widehat{\boldsymbol{\eta}}_{i}} \mathbf{F}_{i 0}+\mathbf{F}_{i 0}^{T} \mathbf{P}_{\widehat{\boldsymbol{\eta}}_{i}}=-\mathbf{Q}_{\widehat{\boldsymbol{\eta}}_{i}} .
$$

Define an auxiliary error $\mathbf{e}_{i}$ as follows:

$$
\mathbf{e}_{i}=\boldsymbol{\eta}_{i}-\widehat{\boldsymbol{\eta}}_{i}+\mathbf{r}_{i}(\mathbf{x}),
$$

and derivative of (17) along with (6), (12), and (15) is given by

$$
\begin{aligned}
\dot{\mathbf{e}}_{i}= & \dot{\boldsymbol{\eta}}_{i}-\dot{\hat{\boldsymbol{\eta}}}_{i}+\frac{\partial \mathbf{r}_{i}(\mathbf{x})}{\partial \mathbf{x}}\left(\mathbf{f}_{i}(\mathbf{x})+\mathbf{g}_{i}(\mathbf{x})\left(\mathbf{u}_{i}-\mathbf{v}_{i}(\mathbf{w})\right)\right) \\
= & \mathbf{F}_{i} \boldsymbol{\eta}_{i}+\mathbf{G}_{i} \gamma_{i}\left(\mathbf{J}_{i} \boldsymbol{\eta}_{i}\right)-\left(\mathbf{F}_{i}-\mathbf{K}_{i} \mathbf{H}_{i}\right)\left(\widehat{\boldsymbol{\eta}}_{i}-\mathbf{r}_{i}(\mathbf{x})\right) \\
& -\mathbf{G}_{i} \gamma_{i}\left(\mathbf{J}_{i}\left(\widehat{\boldsymbol{\eta}}_{i}-\mathbf{r}_{i}(\mathbf{x})\right)\right)-\mathbf{K}_{i} \mathbf{u}_{i}-\frac{\partial \mathbf{r}_{i}(\mathbf{x})}{\partial \mathbf{x}} \mathbf{f}_{i}(\mathbf{x}) \\
= & \mathbf{F}_{i 0} \mathbf{e}_{i}+\mathbf{G}_{i}\left(\gamma_{i}\left(\mathbf{J}_{i} \boldsymbol{\eta}_{i}\right)-\gamma_{i}\left(\mathbf{J}_{i}\left(\boldsymbol{\eta}_{i}-\mathbf{e}_{i}\right)\right)\right) .
\end{aligned}
$$

\section{State Feedback Controller Design}

In terms of the nonlinear internal models shown in (15) and Assumption 1, the state feedback controller can be designed as follows:

$$
\mathbf{u}_{i}=\boldsymbol{\alpha}_{i}(\mathbf{x})+\mathbf{H}_{i}\left(\widehat{\boldsymbol{\eta}}_{i}-\mathbf{r}_{i}(\mathbf{x})\right)
$$

where $\boldsymbol{\alpha}_{i}(\mathbf{x})$ is a controller being able to stabilize the nominal system (8).

Construct a Lyapunov function as follows:

$$
W=V(\mathbf{x})+\sum_{i=1}^{m} \mathbf{e}_{i}^{T} \mathbf{P}_{\widehat{\boldsymbol{\eta}}_{i}} \mathbf{e}_{i} .
$$

Derivative of Lyapunov function $W$ along with system (6) and auxiliary error (18), we can obtain

$$
\begin{aligned}
& \dot{W}=\frac{\partial V(\mathbf{x})}{\partial \mathbf{x}}\left(\mathbf{f}(\mathbf{x})+\sum_{i=1}^{m} \mathbf{g}_{i}(\mathbf{x})\left(\mathbf{u}_{i}-\mathbf{v}_{i}(\mathbf{w})\right)\right) \\
& +\sum_{i=1}^{m} \mathbf{e}_{i}^{T}\left(\left(\mathbf{P}_{\widehat{\boldsymbol{\eta}}_{i}} \mathbf{F}_{i 0}+\mathbf{F}_{i 0}^{T} \mathbf{P}_{\widehat{\boldsymbol{\eta}}_{i}}\right) \mathbf{e}_{i}\right. \\
& \left.+2 \mathbf{e}_{i}^{T} \mathbf{P}_{\widehat{\boldsymbol{\eta}}_{i}} \mathbf{G}_{i}\left(\gamma_{i}\left(\mathbf{J}_{i} \boldsymbol{\eta}_{i}\right)-\gamma_{i}\left(\mathbf{J}_{i}\left(\boldsymbol{\eta}_{i}-\mathbf{e}_{i}\right)\right)\right)\right) \\
& =\frac{\partial V(\mathbf{x})}{\partial \mathbf{x}}\left(\mathbf{f}_{i}(\mathbf{x})+\sum_{i=1}^{m} \mathbf{g}_{i}(\mathbf{x}) \boldsymbol{\alpha}_{i}(\mathbf{x})\right) \\
& +\frac{\partial V(\mathbf{x})}{\partial \mathbf{x}} \sum_{i=1}^{m} \mathbf{g}_{i}(\mathbf{x}) \mathbf{H}_{i}\left(\widehat{\boldsymbol{\eta}}_{i}-\mathbf{r}_{i}(\mathbf{x})\right) \\
& -\frac{\partial V(\mathbf{x})}{\partial \mathbf{x}} \sum_{i=1}^{m} \mathbf{g}_{i}(\mathbf{x}) \mathbf{H}_{i}\left(\mathbf{e}_{i}+\widehat{\boldsymbol{\eta}}_{i}-\mathbf{r}_{i}(\mathbf{x})\right)-\sum_{i=1}^{m} \mathbf{e}_{i}^{T} \mathbf{Q}_{\widehat{\boldsymbol{\eta}}_{i}} \mathbf{e}_{i} \\
& +\sum_{i=1}^{m} 2 \mathbf{e}_{i}^{T} \mathbf{P}_{\widehat{\boldsymbol{\eta}}_{i}} \mathbf{G}_{i}\left(\gamma_{i}\left(\mathbf{J}_{i} \boldsymbol{\eta}_{i}\right)-\gamma_{i}\left(\mathbf{J}_{i}\left(\boldsymbol{\eta}_{i}-\mathbf{e}_{i}\right)\right)\right) \\
& \leq \frac{\partial V(\mathbf{x})}{\partial \mathbf{x}}\left(\mathbf{f}_{i}(\mathbf{x})+\sum_{i=1}^{m} \mathbf{g}_{i}(\mathbf{x}) \boldsymbol{\alpha}_{i}(\mathbf{x})\right) \\
& -\sum_{i=1}^{m} \frac{\partial V(\mathbf{x})}{\partial \mathbf{x}} \mathbf{g}_{i}(\mathbf{x}) \mathbf{H}_{i} \mathbf{e}_{i}-\sum_{i=1}^{m} \lambda_{\min }\left(\mathbf{Q}_{\widehat{\boldsymbol{\eta}}_{i}}\right)\left\|\mathbf{e}_{i}\right\|^{2} \\
& +\sum_{i=1}^{m} 2 \mathbf{e}_{i}^{T} \mathbf{P}_{\widehat{\boldsymbol{\eta}}_{i}} \mathbf{G}_{i}\left(\gamma_{i}\left(\mathbf{J}_{i} \boldsymbol{\eta}_{i}\right)-\gamma_{i}\left(\mathbf{J}_{i}\left(\boldsymbol{\eta}_{i}-\mathbf{e}_{i}\right)\right)\right) \\
& \leq-d_{0}(\|\mathbf{x}\|)+\sum_{i=1}^{m}\left|\frac{\partial V_{i}(\mathbf{x})}{\partial \mathbf{x}} \mathbf{g}_{i}(\mathbf{x})\right|\left\|\mathbf{H}_{i} \mathbf{e}_{i}\right\| \\
& -\sum_{i=1}^{m} \lambda_{\min }\left(\mathbf{Q}_{\hat{\boldsymbol{\eta}}_{i}}\right)\left\|\mathbf{e}_{i}\right\|^{2} \\
& +\sum_{i=1}^{m} 2 \mathbf{e}_{i}^{T} \mathbf{P}_{\widehat{\boldsymbol{\eta}}_{i}} \mathbf{G}_{i}\left(\gamma_{i}\left(\mathbf{J}_{i} \boldsymbol{\eta}_{i}\right)-\gamma_{i}\left(\mathbf{J}_{i}\left(\boldsymbol{\eta}_{i}-\mathbf{e}_{i}\right)\right)\right),
\end{aligned}
$$

where $\lambda_{\min }(\cdot)$ denotes the minimum eigenvalue of a certain matrix. hence

In terms of Assumption 6, we can obtain $\mathbf{P}_{\widehat{\boldsymbol{\eta}}_{i}} \mathbf{G}_{i}=-\left(\mathbf{J}_{i}\right)^{T}$;

$$
\begin{aligned}
& \sum_{i=1}^{m} 2 \mathbf{e}_{i}^{T} \mathbf{P}_{\widehat{\boldsymbol{\eta}}_{i}} \mathbf{G}_{i}\left(\gamma_{i}\left(\mathbf{J}_{i} \boldsymbol{\eta}_{i}\right)-\gamma_{i}\left(\mathbf{J}_{i}\left(\boldsymbol{\eta}_{i}-\mathbf{e}_{i}\right)\right)\right) \\
& =\sum_{i=1}^{m}\left(-2\left(\mathbf{J}_{i} \boldsymbol{\eta}_{i}-\mathbf{J}_{i}\left(\boldsymbol{\eta}_{i}-\mathbf{e}_{i}\right)\right)\right)^{T}\left(\gamma_{i}\left(\mathbf{J}_{i} \boldsymbol{\eta}_{i}\right)-\gamma_{i}\left(\mathbf{J}_{i}\left(\boldsymbol{\eta}_{i}-\mathbf{e}_{i}\right)\right)\right) \leq 0 .
\end{aligned}
$$


Applying permanent establishment inequality $2 a b \leq$ $c a^{2}+c^{-1} b^{2}$ (choosing $c=2$ ) to the second term of (21), we obtain

$$
\begin{aligned}
\sum_{i=1}^{m}\left|\frac{\partial V_{i}(\mathbf{x})}{\partial \mathbf{x}} \mathbf{g}_{i}(\mathbf{x})\right|\left\|\mathbf{H}_{i} \mathbf{e}_{i}\right\| \\
\quad \leq \sum_{i=1}^{m}\left(\left|\frac{\partial V_{i}(\mathbf{x})}{\partial \mathbf{x}} \mathbf{g}_{i}(\mathbf{x})\right|^{2}+\frac{1}{4}\left\|\mathbf{H}_{i}\right\|^{2}\left\|\mathbf{e}_{i}\right\|^{2}\right) .
\end{aligned}
$$

Substitute (22) and (23) into (21) and combine (23) with the application of Assumption 1; we obtain

$$
\dot{W} \leq-\sum_{i=1}^{m}\left(\lambda_{\min }\left(\mathbf{Q}_{\widehat{\boldsymbol{\eta}}_{i}}\right)-\frac{1}{4}\left\|\mathbf{H}_{i}\right\|^{2}\right)\left\|\mathbf{e}_{i}\right\|^{2} .
$$

Choose appropriate $\mathbf{Q}_{\widehat{\boldsymbol{\eta}}_{i}}$ and $\mathbf{H}_{i}$ to satisfy

$$
d_{i}=\lambda_{\min }\left(\mathbf{Q}_{\widehat{\boldsymbol{\eta}}_{i}}\right)-\frac{1}{4}\left\|\mathbf{H}_{i}\right\|^{2}>0 ;
$$

namely,

$$
\dot{W} \leq-\sum_{i=1}^{m} d_{i}\left\|\mathbf{e}_{i}\right\|^{2}
$$

By above knowable, all the variables are bounded. Combine with the application of the invariant set theorem, it can be obtained that $\lim _{t \rightarrow \infty} \mathbf{x}(t)=0$ and $\lim _{t \rightarrow \infty} \mathbf{e}_{i}=0$. Therefore, we give a theorem as follows.

Theorem 7. There exist positive definite matrices $\mathbf{P}_{\widehat{\eta}_{i}}$ and $\mathbf{Q}_{\widehat{\boldsymbol{\eta}}_{i}}$ satisfying (13) and (16), nonzero vector $\mathbf{K}_{i} \in \mathbf{R}^{q}$ makes $\mathbf{F}_{i 0}=$ $\mathbf{F}_{i}-\mathbf{K}_{i} \mathbf{H}_{i}$ be Hurwitz, and (25) holds as well. Hence, for the multivariable nonlinear system (6) and multiclass exosystem (7) satisfying Assumption 1 to Assumption 6, the multiclass nonlinear internal models (15) and control inputs (19) are able to make the close-loop system globally uniformly hounded, and $\lim _{t \rightarrow \infty} \mathbf{x}(t)=0$.

Remark 8. Theorem 7 redescribes Definition 5 in essence; furthermore, it provides a feasible way to find a state feedback controller $\mathbf{u}_{i}$ to stabilize the close-loop system (6) to reference trajectories. In addition, the selection of positive definite matrices $\mathbf{P}_{\widehat{\boldsymbol{\eta}}_{i}}$ and $\mathbf{Q}_{\widehat{\boldsymbol{\eta}}_{i}}$ and nonzero vector $\mathbf{K}_{i}$ is to design multiclass nonlinear internal models (see (15)) to simulate the external nonharmonic disturbances produced by nonlinear exosystem shown in (7). The control inputs (19) are the state feedback controller described in Definition 5.

\section{Numerical Simulation and Analysis}

5.1. Description of Simulation Parameters. The verification of the proposed algorithm in the paper is performed by means of numerical simulation in a $0.018 \mathrm{kWh} / 1.1 \mathrm{~kW}$ PMSM based MEES system. The specific parameters of the MEES system are shown as follows: the rating torque of PMSM $T_{\mathrm{m}}=$ 5.0 N.m, number of pole-pairs $p=4$, flux linkage of rotor $\varphi_{f}=0.18 \mathrm{~Wb}$, resistance of stator $R_{s}=1.95 \Omega$, inductances of $d$-axis and $q$-axis $L_{d}=L_{q}=0.0115 \mathrm{H}$, moment of inertia of rotor $J_{m}=0.008 \mathrm{~kg} \cdot \mathrm{m}^{2}$, ratio of gear case $=40: 1$, the angular velocity of main shaft $\omega_{L}=15 \mathrm{r} / \mathrm{min}$, and damping coefficients of the motor $B_{m}=0.01 \mathrm{~N} / \mathrm{rad} / \mathrm{s}$.

5.2. Analysis and Discussion of Simulation Results. Considering the multiclass nonlinear disturbances, the mathematical model for the whole system of PMSM based MEES system is converted into the form of (6), and the ultimate result is shown in (27). Equation (27) indicates that the nonlinear model of MEES system is a two-variable input system, which is unable to be dealt with by a single-input algorithm. In addition, (27) includes multiclass nonlinear disturbances $\mathbf{v}_{i}(\mathbf{w})$, and the rejection algorithm handling a single disturbance cannot address the problem of multiclass disturbances rejection as well:

$$
\dot{\mathbf{x}}=\mathbf{f}(\mathbf{x})+\sum_{i=1}^{2} \mathbf{g}_{i}(\mathbf{x})\left(\mathbf{u}_{i}-\mathbf{v}_{i}(\mathbf{w})\right),
$$

where $\mathbf{x}=\left[\begin{array}{lll}x_{1} & x_{2} & x_{3}\end{array}\right]^{T}=\left[\begin{array}{lll}i_{d} & \omega_{m} & i_{q}\end{array}\right]^{T}$,

$$
\begin{aligned}
& \mathbf{f}(\mathbf{x})=\left[\begin{array}{c}
-\frac{R_{s}}{L_{d}} x_{1}+p x_{2} x_{3} \\
\frac{p \varphi_{f}}{J_{m}} x_{3}-\frac{B_{m}}{J_{m}} x_{2}-\frac{1}{J_{m}} T_{m} \\
-\frac{R_{s}}{L_{q}} x_{3}-p x_{2} x_{1}-\frac{p \varphi_{f}}{L_{q}} x_{2}
\end{array}\right] \\
& \mathbf{g}_{1}(\mathbf{x})=\left[\begin{array}{ll}
\frac{1}{L_{d}} & 0
\end{array}\right]^{T}, \quad \mathbf{g}_{2}(\mathbf{x})=\left[\frac{1}{L_{q}}\right],
\end{aligned}
$$

and the control input $\mathbf{u}=\left[\begin{array}{ll}u_{1} & u_{2}\end{array}\right]^{T}=\left[\begin{array}{ll}u_{d} & u_{q}\end{array}\right]^{T}$.

If the nonlinear disturbances $\mathbf{v}_{i}(\mathbf{w})$ are ignored in (27), the remaining system in (29) is the nominal system for MEES system:

$$
\dot{\mathbf{x}}=\mathbf{f}(\mathbf{x})+\sum_{i=1}^{2} \mathbf{g}_{i}(\mathbf{x}) \mathbf{u}_{i}
$$

For the sake of convenience, the inputs of nonlinear external disturbances $v_{1}$ and $v_{2}$ are both generated by Van der Pol circuit described in (10) with $\varsigma=2$, which produces bounded limit cycles. Consequently, Assumption 2 holds.

Suppose that $v_{1}$ and $v_{2}$ are immersed in the current components of $d$-axis and $q$-axis, respectively, and $v_{1}=$ $w_{1}, v_{2}=w_{1}-w_{2}$. Hence, the difference of the external disturbances represents the fact that the original system isimmersed in multiclass nonlinear disturbance signals. 
For $v_{1}$, the matrix parameters appearing in (12) are chosen as

$$
\begin{aligned}
& F_{1}=\left[\begin{array}{cc}
2 & 1 \\
-1 & 0
\end{array}\right], \quad G_{1}=\left[\begin{array}{cc}
-2 & 1 \\
0 & 1
\end{array}\right], \quad J_{1}=\left[\begin{array}{cc}
1 & 0 \\
1 & -1
\end{array}\right], \\
& H_{1}=\left[\begin{array}{ll}
1 & 0
\end{array}\right], \quad \gamma_{1}^{1}(s)=\frac{1}{3} s^{3}, \quad \gamma_{1}^{2}(s)=0, \\
& P_{\widehat{\eta}_{1}}=\left[\begin{array}{cc}
\frac{1}{2} & -\frac{3}{2} \\
0 & 1
\end{array}\right] ;
\end{aligned}
$$

for $v_{2}=w_{1}-w_{2}$, the matrix parameters appearing in (12) are selected as

$$
\begin{array}{ll}
F_{2}=\left[\begin{array}{cc}
2 & 1 \\
-1 & 0
\end{array}\right], \quad G_{2}=\left[\begin{array}{cc}
-2 & 1 \\
0 & 1
\end{array}\right], \quad J_{2}=\left[\begin{array}{cc}
1 & 0 \\
1 & -1
\end{array}\right], \\
H_{2}=\left[\begin{array}{cc}
1 & -1
\end{array}\right], \quad \gamma_{2}^{1}(s)=\frac{1}{3} s^{3}, \quad \gamma_{2}^{2}(s)=0, \\
P_{\widehat{\eta}_{2}}=\left[\begin{array}{cc}
\frac{1}{2} & -\frac{3}{2} \\
0 & 1
\end{array}\right],
\end{array}
$$

and hence Assumption 6 is satisfied.

Assume that $c_{1}, c_{2}$, and $c_{3}$ all are certain positive constants; the control law for the nominal system (29) is designed as

$$
\begin{aligned}
\boldsymbol{\alpha}(\mathbf{x})= & {\left[\begin{array}{l}
\boldsymbol{\alpha}_{1}(\mathbf{x}) \\
\boldsymbol{\alpha}_{2}(\mathbf{x})
\end{array}\right] } \\
= & {\left[\begin{array}{c}
-L_{d}\left(c_{1} x_{1}+\frac{L_{q}}{L_{d}} p x_{2} x_{3}\right) \\
L_{q}\left(-\frac{p \varphi_{f}}{J_{m}} c_{2}\left(x_{2}-\omega_{\mathrm{ref}}\right)+\frac{R_{s}}{L_{q}} x_{3}+\frac{L_{d}}{L_{q}} p x_{2} x_{1}\right. \\
+\frac{p \varphi_{f}}{L_{q}} x_{2} \\
\left.-c_{3}\left(x_{3}-\frac{J_{m}}{p \varphi_{f}}\left(\frac{\varphi_{f}}{J_{m}} \omega_{\mathrm{ref}}+\frac{1}{J_{m}} T_{m}\right)\right)\right)
\end{array}\right], }
\end{aligned}
$$

where $\omega_{\text {ref }}$ denotes the angular velocity reference of rotor for PMSM. It can be verified that $\boldsymbol{\alpha}(\mathbf{x})$ can stabilize the nominal system (29) without disturbances, owing to the fact that the stabilization process of (29) is not the emphasis of the paper; hence the detailed deductions are omitted.

Let

$$
\begin{aligned}
\mathbf{V}(\mathbf{x})= & \frac{1}{2} x_{1}^{2}+\frac{1}{2} c_{2}\left(x_{2}-\omega_{\mathrm{ref}}\right)^{2} \\
& +\frac{1}{2}\left(x_{3}-\frac{J_{m}}{p \varphi_{f}}\left(\frac{\varphi_{f}}{J_{m}} \omega_{\mathrm{ref}}+\frac{1}{J_{m}} T_{m}\right)\right)^{2}
\end{aligned}
$$

after calculations and arrangements, we obtain

$$
\begin{aligned}
\frac{\partial \mathbf{V}(\mathbf{x})}{\partial \mathbf{x}}\left(\mathbf{f}(\mathbf{x})+\sum_{i=1}^{2} \mathbf{g}_{i}(\mathbf{x}) \boldsymbol{\alpha}_{i}\right) & \\
= & -\left(c_{1}+\frac{R_{s}}{L_{d}}\right) x_{1}^{2}-\frac{\varphi_{f}}{J_{m}} c_{2}\left(x_{2}-\omega_{\mathrm{ref}}\right)^{2} \\
& -c_{3}\left(x_{3}-\frac{J_{m}}{p \varphi_{f}}\left(\frac{\varphi_{f}}{J_{m}} \omega_{\mathrm{ref}}+\frac{1}{J_{m}} T_{m}\right)\right)^{2} \\
= & -\left(c_{1}+169.5652\right) x_{1}^{2}-225 c_{2}\left(x_{2}-\omega_{\mathrm{ref}}\right)^{2} \\
& -c_{3}\left(x_{3}-\frac{J_{m}}{p \varphi_{f}}\left(\frac{\varphi_{f}}{J_{m}} \omega_{\mathrm{ref}}+\frac{1}{J_{m}} T_{m}\right)\right)^{2}, \\
\frac{\partial \mathbf{V}(\mathbf{x})}{\partial \mathbf{x}} \sum_{i=1}^{2} \mathbf{g}_{i}(\mathbf{x}) & \frac{1}{L_{d}} x_{1}+\frac{1}{L_{q}}\left(x_{3}-\frac{J_{m}}{p \varphi_{f}}\left(\frac{\varphi_{f}}{J_{m}} \omega_{\mathrm{ref}}+\frac{1}{J_{m}} T_{m}\right)\right) \\
= & 86.9565 x_{1}+86.9565\left(x_{3}-\frac{J_{m}}{p \varphi_{f}}\left(\frac{\varphi_{f}}{J_{m}} \omega_{\mathrm{ref}}+\frac{1}{J_{m}} T_{m}\right)\right) .
\end{aligned}
$$

Supposing that

$$
\mathbf{x}^{\prime}=\left[x_{1}\left(x_{2}-\omega_{\mathrm{ref}}\right)\left(x_{3}-\frac{J_{m}}{p \varphi_{f}}\left(\frac{\varphi_{f}}{J_{m}} \omega_{\mathrm{ref}}+\frac{1}{J_{m}} T_{m}\right)\right)\right]^{T},
$$

according to (33), (34), and (35), and choosing $c_{1}=8000$, $c_{2}=40$, and $c_{3}=8000$, we obtain

$$
\begin{gathered}
\frac{1}{2}\left\|\mathbf{x}^{\prime}\right\|^{2} \leq \mathbf{V}(\mathbf{x}) \leq 20\left\|\mathbf{x}^{\prime}\right\|^{2}, \\
\frac{\partial \mathbf{V}(\mathbf{x})}{\partial \mathbf{x}}\left(\mathbf{f}(\mathbf{x})+\sum_{i=1}^{2} \mathbf{g}_{i}(\mathbf{x}) \boldsymbol{\alpha}_{i}\right) \leq-7562\left\|\mathbf{x}^{\prime}\right\|^{2}, \\
\left|\frac{\partial \mathbf{V}(\mathbf{x})}{\partial \mathbf{x}} \sum_{i=1}^{m} \mathbf{g}_{i}(\mathbf{x})\right|^{2} \leq 7562\left\|\mathbf{x}^{\prime}\right\|^{2} .
\end{gathered}
$$

Hence, Assumption 1 holds.

Choose

$$
r_{1}(\mathbf{x})=\left[\begin{array}{ll}
9 L_{d} x_{1} & 0
\end{array}\right]^{T}, \quad r_{2}(\mathbf{x})=\left[\begin{array}{lll}
9 L_{q} x_{3} & 0
\end{array}\right]^{T}
$$

Consequently,

$$
\begin{aligned}
& K_{1}=\frac{\partial r_{1}(\mathbf{x})}{\partial \mathbf{x}} \mathbf{g}_{1}(\mathbf{x})=\left[\begin{array}{ll}
9 & 0
\end{array}\right]^{T}, \\
& K_{2}=\frac{\partial r_{2}(\mathbf{x})}{\partial \mathbf{x}} \mathbf{g}_{2}(\mathbf{x})=\left[\begin{array}{ll}
9 & 0
\end{array}\right]^{T} .
\end{aligned}
$$




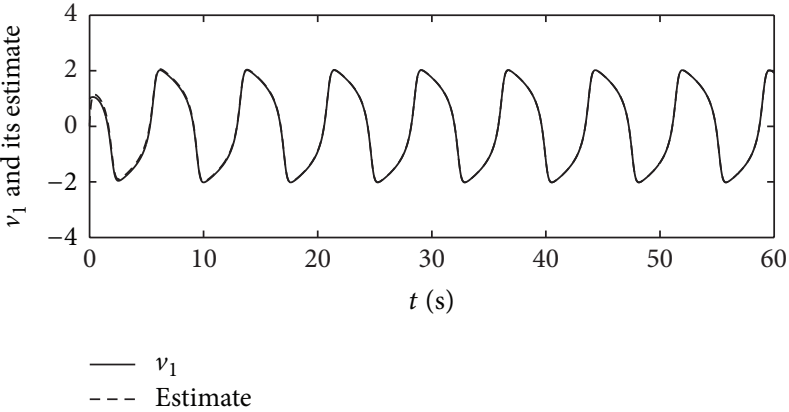

(a)

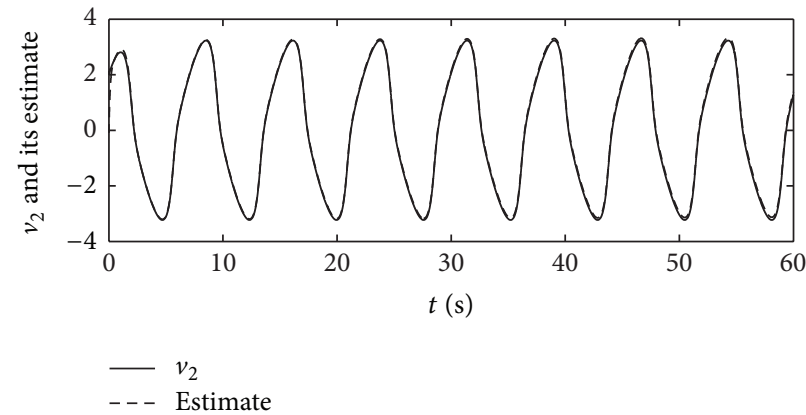

(b)

Figure 2: Nonlinear disturbance inputs $v$ and their estimates.

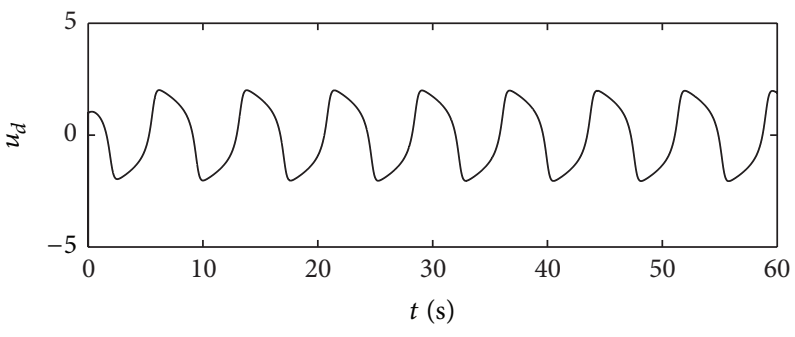

(a)

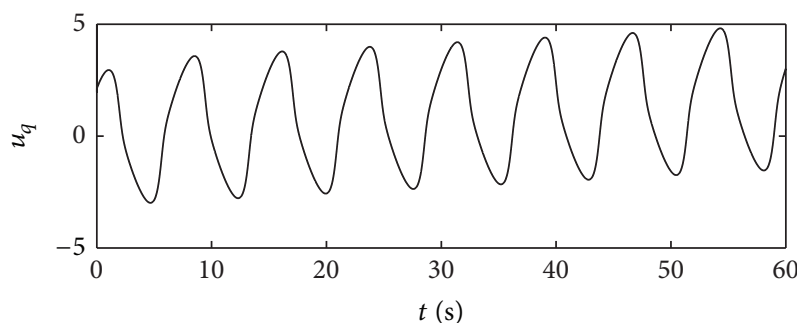

(b)

FIGURE 3: System control inputs $u$.

Therefore, Assumption 4 is satisfied. In addition, with the application of (41), $\mathbf{F}_{i 0}$ and $\mathbf{Q}_{\hat{\eta}_{i}}$ in (16) can be shown as follows by means of some calculations:

$$
\begin{array}{cc}
F_{10}=\left[\begin{array}{ll}
-7 & 1 \\
-1 & 0
\end{array}\right], & Q_{\widehat{\eta}_{1}}=\left[\begin{array}{cc}
\frac{11}{2} & -10 \\
\frac{1}{2} & \frac{3}{2}
\end{array}\right], \\
F_{20}=\left[\begin{array}{cc}
-7 & 10 \\
-1 & 0
\end{array}\right], & Q_{\hat{\eta}_{2}}=\left[\begin{array}{cc}
\frac{11}{2} & -\frac{29}{2} \\
-4 & 15
\end{array}\right] .
\end{array}
$$

The above analysis has verified that MEES system (27) and system (10) of the external disturbances satisfy all the conditions required by Theorem 7. Consequently, based on the multivariable disturbances rejection algorithm proposed in the paper, the multiclass nonlinear internal models and state feedback controller are designed as follows:

$$
\begin{aligned}
\dot{\vec{\eta}}_{1}= & -7 \widehat{\eta}_{1}+10 \widehat{\eta}_{2}-16.8255 x_{1}+0.414 x_{2} x_{3} \\
& -\frac{2}{3}\left(\widehat{\eta}_{1}-0.1035 x_{1}\right)^{3}+9 u_{1}, \\
\dot{\vec{\eta}}_{2}= & -\widehat{\eta}_{1}+0.1035 x_{1}, \\
\dot{\hat{\eta}}_{3}= & -7 \widehat{\eta}_{3}+\widehat{\eta}_{4}-16.8255 x_{3}-6.48 x_{2}-0.414 x_{2} x_{1} \\
& -\frac{2}{3}\left(\widehat{\eta}_{3}-0.1035 x_{3}\right)^{3}+9 u_{2},
\end{aligned}
$$

$$
\begin{aligned}
\dot{\hat{\eta}}_{4}= & -\widehat{\eta}_{3}+0.1035 x_{3}, \\
u_{1}= & -0.2185 x_{1}-0.046 x_{2} x_{3}+\widehat{\eta}_{1}, \\
u_{2}= & -90.1535 x_{3}+247251.84+0.046 x_{2} x_{1} \\
& -413.28 x_{2}+127.7788 T_{m}+\widehat{\eta}_{3}-\widehat{\eta}_{4} .
\end{aligned}
$$

The numerical simulations are conducted in Matlab environment. The whole simulation time is set as $60 \mathrm{~s}$ with the sampling interval $0.001 \mathrm{~s}$; let the initial condition of the simulation be $x(0)=\left[\begin{array}{lll}0.1 & 0 & 1.0\end{array}\right], \widehat{\eta}(0)=\left[\begin{array}{llll}0 & 0 & 0 & 0\end{array}\right]$, and $w(0)=\left[\begin{array}{ll}1 & -1\end{array}\right]$. The reference values of the $d$-axis current $i_{d}$ and angular velocity of the motor $\omega_{m}$ are selected as 0 and $600 \mathrm{r} / \mathrm{min}$, respectively. The $q$-axis current $i_{q}$ tracks the change of the torque of STS in energy storage. The simulation results are shown in Figures 2, 3, and 4. Figure 2 demonstrates the multiclass nonlinear disturbance inputs and their estimations, from which it can be seen that the multiclass nonlinear disturbances acting on the different state variables in a multivariable input system are successfully estimated relying on the internal models designed. Figure 3 displays the control inputs of the system in $d q$ axis under the existence of multiclass nonlinear external disturbances. Figure 4 describes the system states, which indicates that the system achieves the asymptotical tracking for the reference signals rapidly and the multiclass nonlinear disturbances are completely suppressed. Hence, the multivariable controller designed in the paper has a good control performance. 


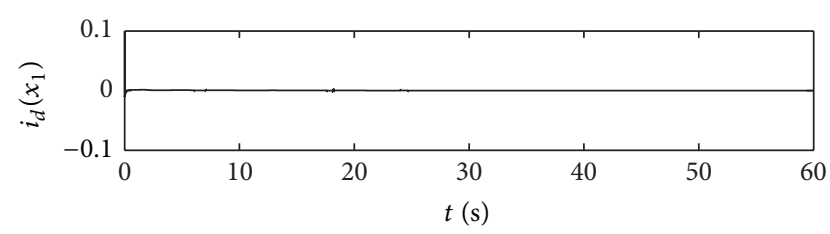

(a)

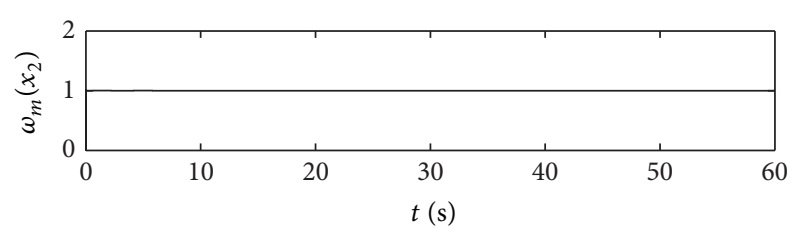

(b)

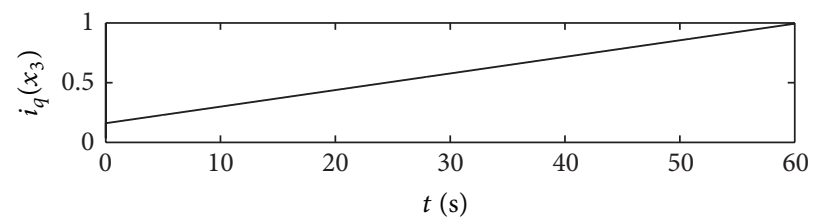

(c)

Figure 4: System states $x$.

\section{Conclusion}

In light of the strong coupling and nonlinear characteristics of PMSM based MEES system, a global multiclass nonharmonic disturbances rejection method for general multivariable nonlinear system under multiclasses nonlinear exosystems is proposed in the paper. For multiclass nonlinear external disturbances with different periodic bounded nonharmonic characteristics, different nonlinear internal model equations are designed. Based on design of control law for nominal system, a state feedback controller for original system is presented and a Lyapunov function is established to theoretically testify the global boundedness of all signals in multivariable close-loop system. The simulation results show that the multiclass different nonlinear disturbance inputs are all completely rejected and the close-loop system can track the reference signals promptly. Consequently, high accuracy servo control for PMSM based MEES system is realized.

In addition to PMSM based MEES system, many other practical engineering systems, including turbine motor, generator, power flexible manipulator, and communication circuit, are frequently affected by the nonharmonic disturbances generated by the external nonlinear exosystems. As the most famous and typical nonlinear circuit, Van der Pol circuit researched in the paper will excite nonharmonic disturbances and make the system mentioned above produce nonharmonic forced oscillation. The algorithm presented in the paper can eliminate the harmful oscillation and improve the stability for these practical systems.

The critical points of the output regulation problem under nonharmonic disturbances are to model the nonlinear exosystems and propose reasonable algorithm to stabilize the closed-loop system. In the future, the proposed algorithm in the paper can be able to be extended to uncertainly multivariable systems and unknown external signals; correspondingly, the innovative control technologies should be researched to cope with the more complex and generalized circumstances.

\section{Conflict of Interests}

The authors declare that there is no conflict of interests regarding the publication of this paper.

\section{Acknowledgments}

This work was supported by the National Natural Science Foundation of China (Grant no. 51077053), the Specialized Research Fund for the Doctoral Program of Higher Education of China (Grant no. 20120036130001), the Fundamental Research Funds for the Central Universities of China (Grant no. 2014MS93), and the Independent Research Funds of State Key Laboratory of Alternate Electrical Power System with Renewable Energy Sources of China (Grant no. 201209).

\section{References}

[1] H. Chen, T. N. Cong, W. Yang, C. Tan, Y. Li, and Y. Ding, "Progress in electrical energy storage system: a critical review," Progress in Natural Science, vol. 19, no. 3, pp. 291-312, 2009.

[2] H. Ibrahim, A. Ilinca, and J. Perron, "Energy storage systemsCharacteristics and comparisons," Renewable and Sustainable Energy Reviews, vol. 12, no. 5, pp. 1221-1250, 2008.

[3] Y. Yu and Z. Q. Mi, "Nonlinear dynamic model and chaotic characteristics of mechanical elastic energy storage unit in energy storage process," Acta Physica Sinica, vol. 62, no. 3, Article ID 038043, 2013.

[4] B. M. Ebrahimi, J. Faiz, M. Javan-Roshtkhari, and A. Z. Nejhad, "Static eccentricity fault diagnosis in permanent magnet synchronous motor using time stepping finite element method," IEEE Transactions on Magnetics, vol. 44, no. 11, pp. 4297-4300, 2008.

[5] Y. Yu and Z. Mi, "Dynamic modeling and control of electromechanical coupling for mechanical elastic energy storage system," Journal of Applied Mathematics, vol. 2013, Article ID 603063, 11 pages, 2013. 
[6] F. Wang and Q. Yu, "Speed-regulation system of PMLSM Based on BP neural network PID control," Applied Mechanics and Materials, vol. 466-467, pp. 1217-1221, 2012.

[7] S. He, "Resilient $L_{2}-L_{\infty}$ filtering of uncertain Markovian jumping systems within the finite-time interval," Abstract and Applied Analysis, vol. 2013, Article ID 791296, 7 pages, 2013.

[8] J. Song and S. He, "Nonfragile robust finite-time $L_{2}-L_{\infty}$ controller design for a class of uncertain Lipschitz nonlinear systems with time-delays," Abstract and Applied Analysis, vol. 2013, Article ID 265473, 9 pages, 2013.

[9] Z.-G. Wu, P. Shi, H. Su, and J. Chu, "Asynchronous $l_{2}-l_{\infty}$ filtering for discrete-time stochastic Markov jump systems with randomly occurred sensor nonlinearities," Automatica, vol. 50, no. 1, pp. 180-186, 2014.

[10] H. Shen, Y. Chu, S. Xu, and Z. Zhang, "Delay-dependent Ho control for jumping delayed systems with two markov processes," International Journal of Control, Automation and Systems, vol. 9, no. 3, pp. 437-441, 2011.

[11] S. He and F. Liu, "Adaptive observer-based fault estimation for stochastic Markovian jumping systems," Abstract and Applied Analysis, vol. 2012, Article ID 176419, 11 pages, 2012.

[12] S. P. He and F. Liu, "Finite-time Ho control of nonlinear jump systems with time-delays via dynamic observer-based state feedback," IEEE Transactions on Fuzzy Systems, vol. 20, no. 4, pp. 605-614, 2012.

[13] L. Paunonen and S. Pohjolainen, "Reduced order internal models in robust output regulation," IEEE Transactions on Automatic Control, vol. 58, no. 9, pp. 2307-2318, 2013.

[14] A. Isidori and C. I. Byrnes, "Output regulation of nonlinear systems," IEEE Transactions on Automatic Control, vol. 35, no. 2, pp. 131-140, 1990.

[15] J. Huang and Z. Chen, "A general framework for tackling the output regulation problem," IEEE Transactions on Automatic Control, vol. 49, no. 12, pp. 2203-2218, 2004.

[16] Z.-K. Li, Z.-S. Duan, and G.-R. Chen, "Disturbance rejection and $\mathrm{H} \infty$ pinning control of linear complex dynamical networks," Chinese Physics B, vol. 18, no. 12, pp. 5228-5234, 2009.

[17] Z. Ding, "Asymptotic rejection of unknown sinusoidal disturbances in nonlinear systems," Automatica, vol. 43, no. 1, pp. 174177, 2007.

[18] Y. Jiang and S. Liu, "Global disturbance rejection of a class of nonlinear systems with unknown exosystems," Journal of Control Theory and Applications, vol. 7, no. 4, pp. 379-383, 2009.

[19] J. Huang and W. J. Rugh, "On a nonlinear multivariable servomechanism problem," Automatica, vol. 26, no. 6, pp. 963972, 1990.

[20] C. L. Chen, Z. Ding, and B. Lennox, "Rejection of nonharmonic disturbances in nonlinear systems with semi-global stability," IEEE Transaction on Circuits and Systems II: Express Briefs, vol. 55, no. 12, pp. 1289-1293, 2008.

[21] Y. Jiang and S. Liu, "Global disturbance rejection of a class of nonlinear systems with unknown external disturbances," Journal of Shandong University (Engineering Science), vol. 41, no. 1, pp. 49-54, 2011.

[22] S. Liu, Y. Jiang, and P. Liu, "Rejection of nonharmonic disturbances in nonlinear systems," Kybernetika, vol. 46, no. 5, pp. 785-798, 2010.

[23] Y. Yu, Z. Mi, Y. Che, T. Zhao, and Y. Xu, "Global harmonic current rejection of nonlinear backstepping control with multivariable adaptive internal model principle for grid-connected inverter under distorted grid voltage," Mathematical Problems in Engineering, vol. 2013, Article ID 749798, 15 pages, 2013.
[24] G. Zhu, L.-A. Dessaint, O. Akhrif, and A. Kaddouri, "Speed tracking control of a permanent-magnet synchronous motor with state and load torque observer," IEEE Transactions on Industrial Electronics, vol. 47, no. 2, pp. 346-355, 2000.

[25] Z. Ping and J. Huang, "Global robust output regulation for a class of multivariable systems and its application to a motor drive system," in Proceedings of the American Control Conference (ACC '11), pp. 4560-4565, San Francisco, Calif, USA, July 2011. 


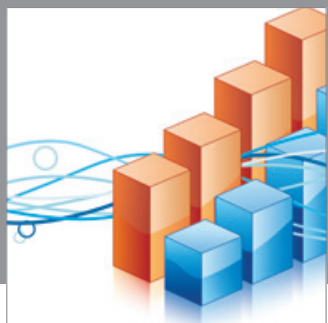

Advances in

Operations Research

mansans

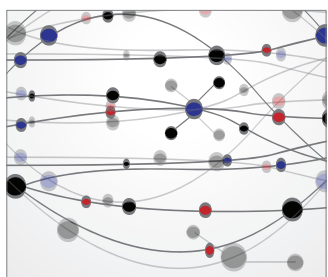

The Scientific World Journal
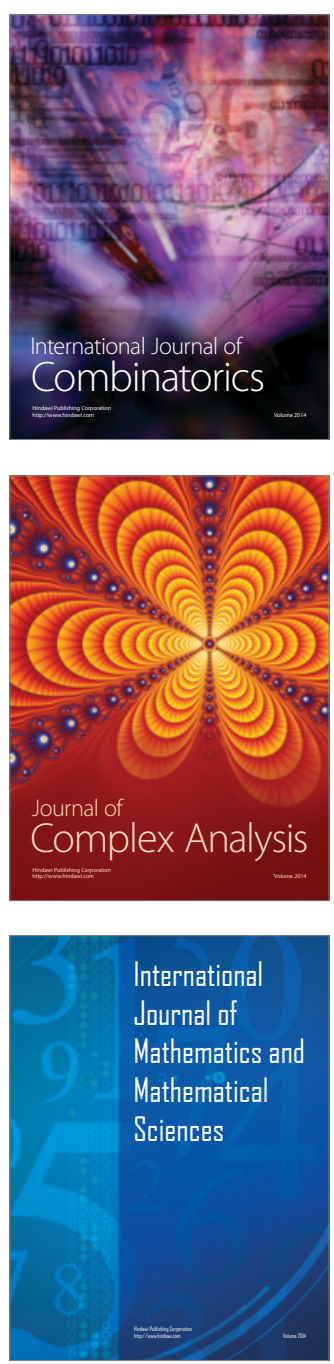
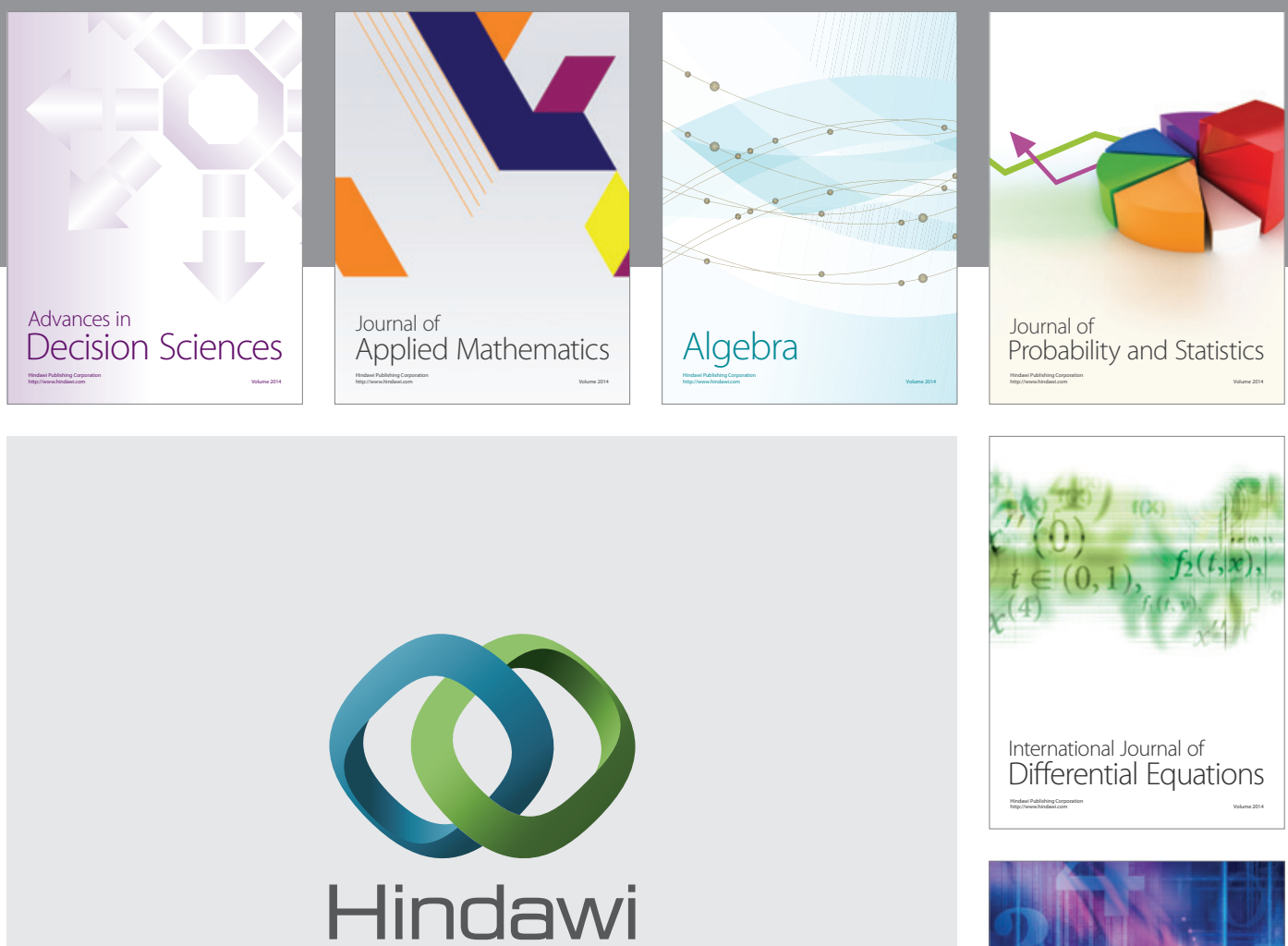

Submit your manuscripts at http://www.hindawi.com
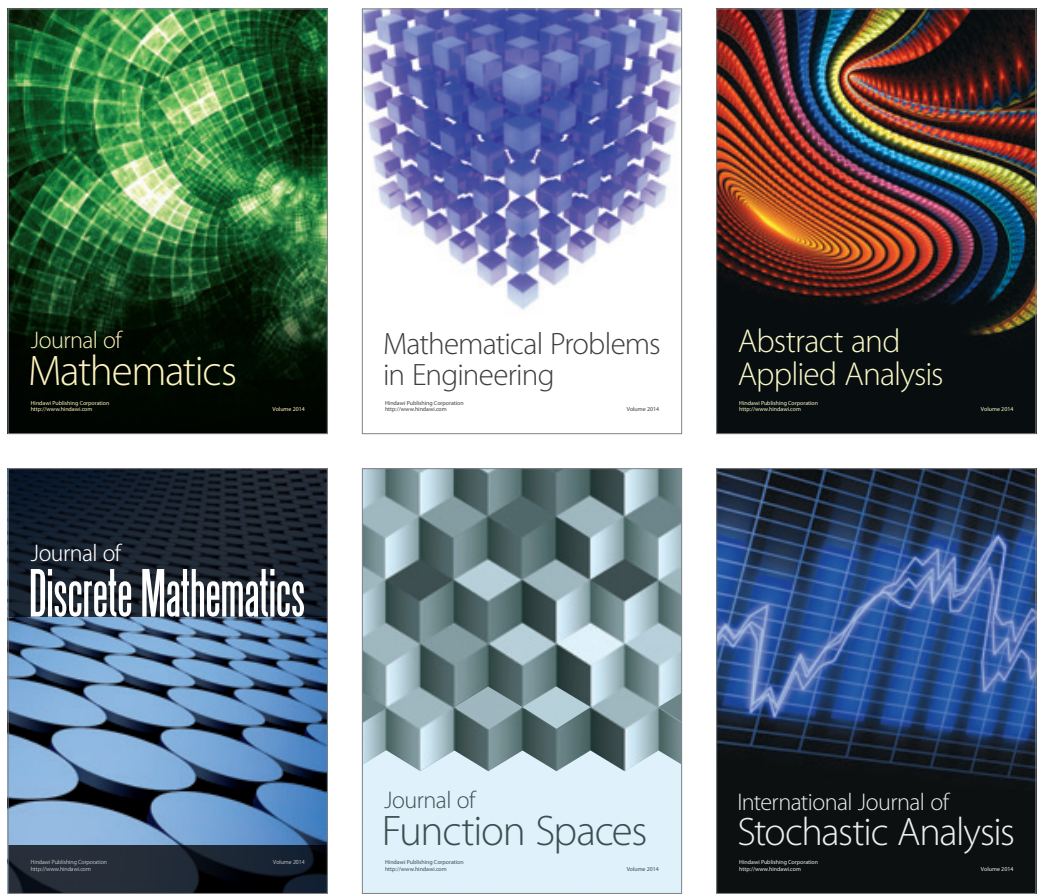

Journal of

Function Spaces

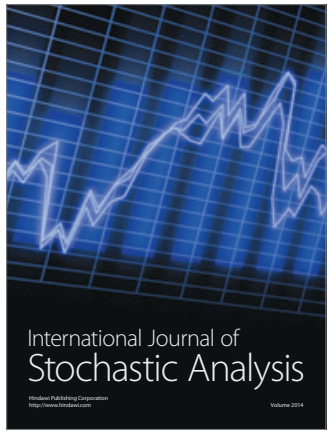

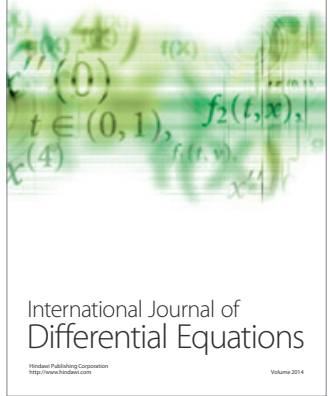
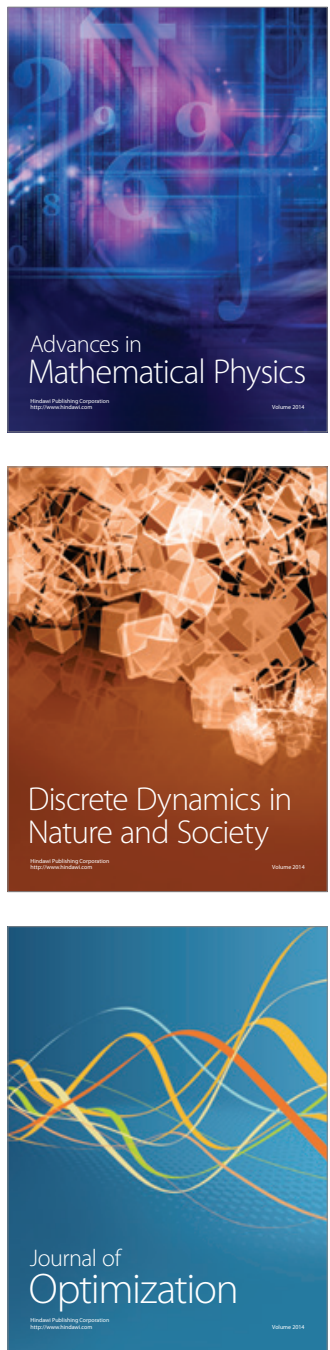